\title{
Correlative X-ray Ptychographic and Fluorescence Imaging at the Advanced Photon Source
}

\author{
Junjing Deng ${ }^{1 *}$, Si Chen ${ }^{1}$, Qiaoling Jin ${ }^{2}$, Everett Vacek ${ }^{2}$, Chris Jacobsen ${ }^{1,2,3}$, Barry Lai ${ }^{1}$ and Stefan Vogt ${ }^{1}$ \\ ${ }^{1}$ Advanced Photon Source, Argonne National Lab, Lemont, IL 60439, USA \\ ${ }^{2}$ Department of Physics and Astronomy, Northwestern University, Evanston, IL 60208, USA \\ ${ }^{3}$ Chemistry of Life Processes Institute, Northwestern University, Evanston, IL 60208, USA \\ * Corresponding author: junjingdeng@anl.gov
}

Accurate knowledge of elemental distributions of specimens within their structural context is very important for understanding their roles and the resulting sample behaviors. Recent developed techniques in visible light fluorescence microscopy provide high-resolution views of samples [1], but only image certain preselected cell's components due to the dependence on binding affinities. Electron microscopy is able to provide high-resolution information down to the nanoscale, and its combination with a light microscope called Correlative Light and Electron Microscopy (CLEM) has been a success of correlative microscopy as described in a recent review [2]. However, due to the extremely low penetration of electrons, it can only image either exposed cellular surfaces in scanning electron microscope, or thin samples (usually $<1 \mu \mathrm{m}$ ) in transmission type.

X-ray microscopy bridges the gap between light and electron microscopy. With its high penetration depth, X-ray microscopy can examine thick and unstained biological specimens that are difficult to observe in transmission electron or light microscopy, offering different imaging modalities by means of fluorescence, absorption, and phase shift. At the Advanced Photon Source (APS), we have developed a correlative imaging method via a combination of X-ray fluorescence microscopy (XFM) and ptychography $[3,4,5]$. The characteristic fluorescence of chemical elements produced by X-rays removes the need to add or genetically encode specific fluorescence labels into the specimens. With nanofocusing optics such as Fresnel zone plates, XFM is able to achieve nanometer-scale resolution nowadays. Ptychography, a scanning version of coherent diffractive imaging method, can achieve higher spatial resolution with no limitation from the focusing beam size. Moreover, both quantitative absorption and phase contrast can be obtained simultaneously in ptychography. Since both XFM and ptychography are operated in the same scanning mechanism, they can be perfectly integrated into one experiment for simultaneous multi-modal imaging. This simultaneous data acquisition streamlines the experiment and saves considerable amount of time by avoiding sample transfer and registration between different instruments, and thus reduces the radiation dose on the sample.

At the APS, two scanning microscopes have been developed for correlative ptychography and fluorescence imaging. The micro-fluorescence probe at Sector 2-ID-D focuses X-ray beam using zone plates to provide a spatial resolution of $150-300 \mathrm{~nm}$ for elemental mapping. The extension of ptychography is realized by placing a pixel area detector downstream of the sample to collect far-field diffraction patterns which are then used to reconstruct high-resolution sample structure. Ptychography imaging on this instrument typically achieves sub-30 nm spatial resolution. This microprobe is operated in atmospheric pressure (usually in helium environment to reduce air absorption and scattering). It is suitable for 2D correlative imaging of dehydrated or chemical fixed biological samples and materials samples. To provide a more stable sample environment and to study the biological samples closer to their natural state, a hard X-ray nanoprobe called Bionanoprobe (BNP) has been built providing a high 
vacuum and cryogenic sample environment [6]. Cryogenic conditions keep samples in frozen-hydrated state for high fidelity structural and elemental preservation as well as the mitigation of radiation damage. The BNP routinely operates with sub $100 \mathrm{~nm}$ for fluorescence imaging. The cryogenic sample environment and advanced sample positioning at the BNP allow one to get a spatial resolution of sub 20 $\mathrm{nm}$ for ptychographic imaging [4]. Combined with sample tilting to different orientations for 3D imaging, such correlative methods can provide unprecedented access to the inner working of whole samples with high spatial resolution and multi-modalities of structural and chemical information. We have recently demonstrated 3D correlative imaging method at the BNP on a frozen-hydrated algae cell (Fig. 1) [5]. 3D XFM provides trace element distributions of various organelles within the whole alga. The elemental distributions are coupled with ptychography to reveal complex ultrastructure information.

\section{References:}

[1] SJ Sahl, SW Hell, and S Jakobs, Nat. Rev. Mol. Cell Biol. 18(2017), p. 685-701.

[2] AA Mironov, and GV Beznoussenko, Methods Enzymol. 504 (2012), p. 201-219.

[3] J Deng et al., Proc. Natl. Acad. Sci. U.S.A. 112(2015), p. 2314-2319.

[4] J Deng et al., Sci. Rep. 7 (2017), p. 445.

[5] J Deng et al., Sci. Adv. 4 (2018), p. eaau4548.

[6] S Chen et al., J. Synchrotron Radiat. 21(2014), p. 66-75.

[7] The BNP is funded by NIH/National Center for Research Resources High End Instrumentation Grant 1S10RR029272-01. This research used resources of the Advanced Photon Source, a U.S. Department of Energy (DOE) Office of Science User Facility operated for the DOE Office of Science by Argonne National Laboratory under Contract No. DE-AC02-06CH11357.

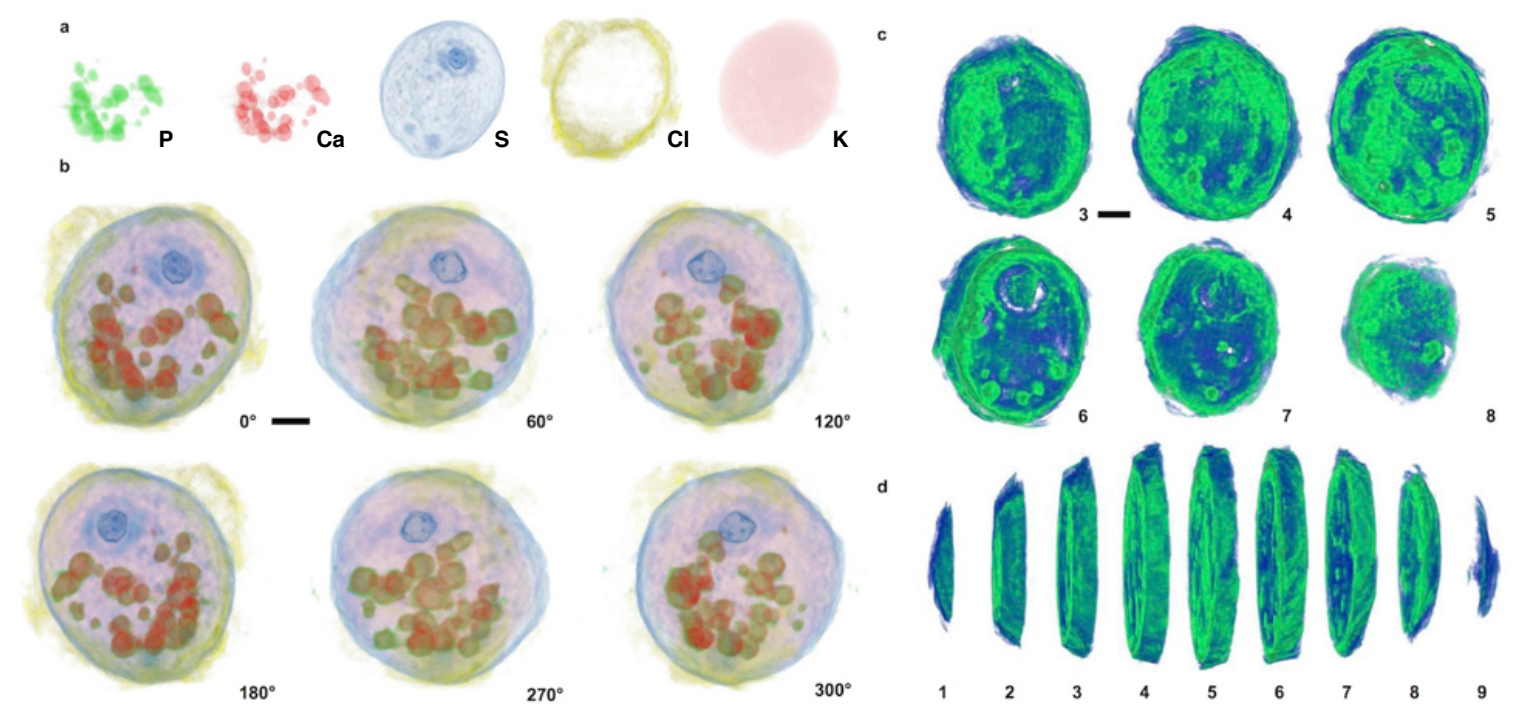

Figure 1. $3 \mathrm{D}$ correlative imaging of a whole frozen-hydrated $C$. reinhardtii alga using X-ray ptychography and tomography. Reconstructed 3D elemental distributions (a) and their composite volume viewed in $60^{\circ}$ increments (b). (c) Slabs of $\sim 2 \mu \mathrm{m}$ thickness through the ptychographic 3D volume. (d) Cut outs of the entire reconstructed volume viewed at $80^{\circ}$ angle. Scale bar, $2 \mu \mathrm{m}$. 